\title{
Application of Image Moment Flow of a RPP to 6 DOF Visual Tracking
}

\author{
Huah $T u^{1}$, Li-Chen $F u^{1,2}$
}

\author{
Department of Electronic Engineering ${ }^{1}$ \\ Department of Computer Science and Information Engineering ${ }^{2}$ \\ National Taiwan University, Taipei, Taiwan, R.O.C. \\ huah@irl.csie.nru.edu.tw,lichen@csie.ntu.edu.tw
}

\begin{abstract}
The image moment flow of a rigid planar patch (RPP) is derived explicitly in this paper. From that derived relation, one can estimate the pose information of the RPP and, further, track a 6 DOF RPP using a properly designed visual feedback control.
\end{abstract}

\section{Introduction}

As the robot is required to perform more and more complex tasks for practical use, the requirement of visual application has gradually changed from the analysis of static visual information to the dynamical visual tracking [1]-[4]. In order to let the visual tracking algorithms easily satisfy the real-time requirement, we have concentrated on finding the characteristics of the image moment of a RPP.

\section{Preliminaries}

In the following description, the frame information will be revealed by the left superscript and the left subscript, and $\{w\},\{c\},\{o\}$ denote the world frame, the camera frame, and the object frame, respectively. The left subsctipt denotes the destination frame, or just the adopted frame, and the left superscript denotes the original frame, or the active frame.

\subsection{Frame Operation}

Under the condition ${ }_{2} P$ is fixed, the following equations are assumed to be well-known:

$$
\begin{aligned}
{ }_{1} P & ={ }_{1}^{2} R_{2} P+{ }_{1}^{2} T, \\
{ }_{1} \dot{P} & ={ }_{1}^{2} \omega \times{ }_{1} P+{ }_{1}^{2} k \\
& ={ }_{1}^{2} \dot{R}_{2} P+{ }_{1}^{2} \dot{T},
\end{aligned}
$$

\subsection{Rigid Planar Patch}

The RPP, $r p p$, is a rigid object which can be entirely contained in a plane. Therefore, we can attach an object frame $\{o\}$ to this object such that

$$
P \in r p p \Rightarrow{ }_{o} P_{z}=0 \text {. }
$$

And, we will let $\left[\begin{array}{c}s \\ t\end{array}\right]=\left[\begin{array}{c}{ }_{o} P_{x} \\ o P_{y}\end{array}\right]$ be the coordinate in the RPP plane. According to above consideration,

${ }_{c} \dot{P}=\left[\begin{array}{l}{ }_{c}^{o} \omega_{y} z-{ }_{c}^{o} \omega_{z} y+q_{1}{ }_{c}^{o} k_{x} x+q_{2}{ }_{c}^{o} k_{x} y+q_{3}{ }_{c}^{o} k_{x} z \\ { }_{c}^{o} \omega_{z} x-{ }_{c}^{o} \omega_{x} z+q_{1}{ }_{c}^{o} k_{y} x+q_{2}{ }_{c}^{o} k_{y} y+q_{3}{ }_{c}^{o} k_{y} z \\ { }_{c}^{o} \omega_{x} y-{ }_{c}^{o} \omega_{y} x+q_{1}{ }_{c}^{o} k_{z} x+q_{2}{ }_{c}^{o} k_{z} y+q_{3}{ }_{c}^{o} k_{z} z\end{array}\right]$,

where $q=\frac{1}{{ }_{\mathrm{c}} R_{3} \cdot{ }_{\mathrm{c}}^{\circ} T}{ }_{c}^{o} R_{3}$.

\subsection{Camera Model}

In this paper, the camera model is assumed to be perspective projection. Then, the transformation from the RPP plane to the camera plane will be

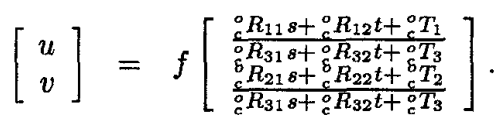

\section{Image Moments of the RPP and Their Flows}

Here, we only discuss the image moments of binary images.

\subsection{Original Image Moment}

The image moment with $(i, j)$-th order is defined by

$$
\begin{aligned}
m_{i j} & =\iint_{(s, t) \in r p p} u(s, t)^{i} v(s, t)^{j} d u d v \\
& ={ }_{c}^{o} R_{3} \cdot{ }_{c}^{o} T \iint \frac{f^{i+j+2}{ }_{c} x^{i}(s, t){ }_{c} y^{j}(s, t)}{{ }_{c} z^{i+j+3}(s, t)} d s d t .
\end{aligned}
$$


From (5), and applying (3) and the definition of $q$, the derivative of the $(i, j)$-th moment is

$$
\begin{aligned}
\dot{m}_{i j}= & \left(-\frac{i+j+3}{f} m_{i(j+1)}-j f m_{i(j-1)}\right){ }_{c}^{o} \omega_{x} \\
& \left(\frac{i+j+3}{f} m_{(i+1) j}+i f m_{(i-1) j}\right){ }_{c}^{o} \omega_{y} \\
& \left(-i m_{(i-1)(j+1)}+j m_{(i+1)(j-1)}\right){ }_{c}^{o} \omega_{z} \\
& \left((i+1) q_{1} m_{i j}+i q_{2} m_{(i-1)(j+1)}\right. \\
& \left.+i f q_{3} m_{(i-1) j}\right){ }_{c}^{o} k_{x} \\
& \left(j q_{1} m_{(i+1)(j-1)}+(j+1) q_{2} m_{i j}\right. \\
& \left.+j f q_{3} m_{i(j-1)}\right){ }_{c}^{o} k_{y} \\
& \left(-\frac{i+j+3}{f} q_{1} m_{(i+1) j}-\frac{i+j+3}{f} q_{2} m_{i(j+1)}\right. \\
& \left.-(i+j+2) q_{3} m_{i j}\right){ }_{c}^{o} k_{z} \\
= & A_{i j}\left(m_{e x t}, q\right)\left[\begin{array}{c}
o \\
c \\
o \\
c \\
c
\end{array}\right]
\end{aligned}
$$

Combining different moment flow equations, we can thus obtain a suitable image moment flow system

$$
\dot{m}=A\left(m_{e x t}, q\right)\left[\begin{array}{c}
o \\
c \\
o \\
c \\
c
\end{array}\right]
$$

where the symbol $m_{\text {ext }}$ means that the matrix $A$ should reference to the moments which do not belong to $m$.

\subsection{Normalized Image Moment}

Sometimes, normalized image moment can lead to better performance, which is defined to be

$$
\bar{m}_{i j}=\frac{m_{i j}}{m_{00}},
$$

then,

$$
\dot{\bar{m}}_{i j}=\bar{A}_{i j}\left(\bar{m}_{e x t}, q\right)\left[\begin{array}{c}
o \\
c \\
o \\
c \\
c
\end{array}\right] .
$$

\subsection{Point Feature}

Point feature can been seen as an RPP with very small area. By simple approximation and reduction, the optical flow equation can be derived.

\section{Moment-Based Visual Track- ing}

Under the dynamical equation (7), the proposed Lyapunov-based control is

$$
\begin{aligned}
& {\left[\begin{array}{c}
o \omega \\
c \\
o \\
c
\end{array}\right] } \\
= & -\pi\left(\left|e^{T} \dot{m}_{d}\right|+\sigma\right) \frac{P A^{t}\left(m_{e x t}, q\right) e}{\left|e^{t} A\left(m_{e x t}, q\right) P A^{t}\left(m_{e x t}, q\right) e\right|+\epsilon}
\end{aligned}
$$

where $e=m-m_{d}, \sigma$ and $\epsilon$ are positive numbers, and $P$ is a positive definite gain matrix.

\section{Simulation Results}

In this simulatin, one of the object's face contains three RPPs. Our objective is to visually track this moving object by moment measurement. From Fig.1, we can see that the moments converge to the desired moments asymptotically.

\section{Conclusion}

In this paper, we developed a new technique to treat with the image moments of RPPs. Through the moment flow equations and properly designed control law, visual tracking can be achieved and the effectiveness was demostrated by the simulation result.
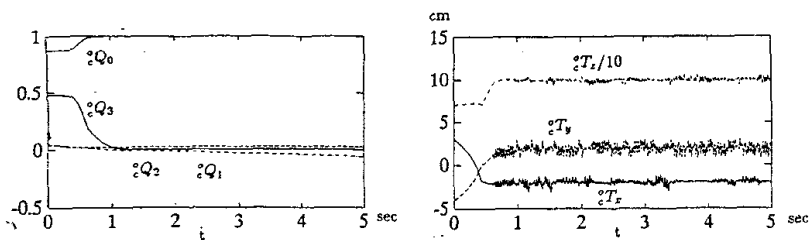

Fig. 1 practical pose trajectory in Example 1

\section{References}

[1] J. T. Feddema and C. S. G. Lee, "Adaptive Image Feature Prediction and Control for Visual Tracking with a Hand-Eye Coordinated Camera," IEEE Trans. on Systems, Man and Cybernetics, vol. 20 , no. 5, pp. 1172-1183, Sept./Oct. 1990.

[2] L. E. Weiss, A. C. Sanderson, and C. P. Neuman, "Dynamic Sensor-Based Control of Robots with Visual Feedback," IEEE J. on Robotics and Automation, vol. RA-3, no. 5, pp. 404-417, 1987.

[3] N. P. Papanikolppoulos, B. Nelson, and P. K. Khosla, "Six Degree-of-Freedom Hand/Eye Visual Tracking with Uncertain Parameters," Proc. IEEE Int. Conf. on Robotics and Automation, pp.174-179, 1994.

[4] J. Y. Aloimonos and D. Tsakiris, "On the Visual Mathematics of Tracking," Image and Vision Computing, vol. 9, no. 4, pp. 235-251, Aug. 1991. 